\title{
Periodicity and Solution of Rational Recurrence Relation of Order Six
}

\author{
Tarek F. Ibrahim ${ }^{1,2}$ \\ ${ }^{1}$ Department of Mathematics, Faculty of Sciences and Arts (S. A.), King Khalid University, Abha, KSA \\ ${ }^{2}$ Department of Mathematics, Faculty of Science, Mansoura University, Mansoura, Egypt \\ Email: tfibrahem@mans.edu.eg
}

Received April 27, 2012; revised May 27, 2012; accepted June 3, 2012

\begin{abstract}
Difference equations or discrete dynamical systems is diverse field whose impact almost every branch of pure and applied mathematics. Every dynamical system $a_{n+1}=f\left(a_{n}\right)$ determines a difference equation and vise versa. We obtain in this paper the solution and periodicity of the following difference equation.

$$
x_{n+1}=\left(x_{n} x_{n-2} x_{n-4}\right) /\left(x_{n-1} x_{n-3} x_{n-5}\right),
$$

$n=0,1, \cdots$ where the initial conditions $x_{-5}, x_{-4}, x_{-3}, x_{-2}, x_{-1}$ and $x_{0}$ are arbitrary real numbers with $x_{-1}, x_{-3}$ and $x_{-5}$ not equal to be zero. On the other hand, we will study the local stability of the solutions of Equation (1). Moreover, we give graphically the behavior of some numerical examples for this difference equation with some initial conditions.
\end{abstract}

Keywords: Difference Equation; Solutions; Periodicity; Local Stability

\section{Introduction}

Difference equations or discrete dynamical systems is diverse field whose impact almost every branch of pure and applied mathematics. Every dynamical system $a_{n+1}=f\left(a_{n}\right)$ determines a difference equation and vise versa. Recently, there has been great interest in studying difference equations. One of the reasons for this is a necessity for some techniques whose can be used in investigating equations arising in mathematical models decribing real life situations in population biology, economic, probability theory, genetics, psychology, ...etc. Difference equations usually describe the evolution of certain phenomenta over the course of time. Recently there are a lot of interest in studying the global attractivity, boundedness character the periodic nature, and giving the solution of nonlinear difference equations. Recently there has been a lot of interest in studying the boundedness character and the periodic nature of nonlinear difference equations. Difference equations have been studied in various branches of mathematics for a long time. First results in qualitative theory of such systems were obtained by Poincaré and Perron in the end of nineteenth and the beginning of twentieth centuries. For some results in this area, see for example [1-13].

Although difference equations are sometimes very simple in their forms, they are extremely difficult to un- derstand throughly the behavior of their solutions.

Many researchers have investigated the behavior of the solution of difference equations for examples.

Cinar [1,2] investigated the solutions of the following difference equations

$$
\begin{aligned}
& x_{n+1}=x_{n-1} /\left(-1+x_{n} x_{n-1}\right) \\
& x_{n+1}=a x_{n-1} /\left(1+b x_{n} x_{n-1}\right)
\end{aligned}
$$

Karatas et al. [4] gave that the solution of the difference equation

$$
x_{n+1}=x_{n-5} /\left(1+x_{n-2} x_{n-5}\right)
$$

G. Ladas, M. Kulenovic et al. [12] have studied period two solutions of the difference equation

$$
x_{n+1}=\left(\alpha+\beta x_{n}+\gamma x_{n-1}\right) /\left(A+B x_{n}+C x_{n-1}\right)
$$

Simsek et al. [13] obtained the solution of the difference equation

$$
x_{n+1}=x_{n-3} /\left(1+x_{n-1}\right)
$$

Ibrahim [5] studied the third order rational difference Equation

$$
x_{n+1}=\left(x_{n} x_{n-2}\right) /\left(x_{n-1}\left(\alpha+\beta x_{n} x_{n-2}\right)\right)
$$

In this paper we obtain the solution and study the periodicity of the following difference equation 


$$
x_{n+1}=\left(x_{n} x_{n-2} x_{n-4}\right) /\left(x_{n-1} x_{n-3} x_{n-5}\right),
$$

$n=0,1, \cdots$ where the initial conditions $x_{-5}, x_{-4}, x_{-3}$, $x_{-2}, x_{-1}$, and $x_{0}$ are arbitrary real numbers with $x_{-1}$, $x_{-3}$ and $x_{-5}$ not equal to be zero. On the other hand, we will study the local stability of the solutions of Equation (1). Moreover, we give graphically the behavior of some numerical examples for this difference equation with some initial conditions.

Here, we recall some notations and results which will be useful in our investigation.

Let $I$ be some interval of real numbers and Let $F: I^{k+1} \rightarrow I$ be a continuously differentiable function. Then for every set of initial conditions $x_{-k}, x_{-k+1}, \cdots$, $x_{0} \in I$, the difference equation

$$
x_{n+1}=F\left(x_{n}, x_{n-1}, \cdots, x_{n-k}\right), n=0,1, \cdots,
$$

has a unique solution $\left\{x_{n}\right\}_{n=-k}^{\infty}[11]$.

Definition (1.1) A point $\bar{x} \in I$ is called an equilibrium point of Equation (2) if

$$
\bar{x}=F(\bar{x}, \bar{x}, \cdots, \bar{x}) .
$$

That is, $x_{n}=\bar{x}$ for $n \geq 0$, is a solution of Equation (2), or equivalently, $\bar{x}$ is a fixed point of $F$.

Definition (1.2) The difference Equation (2) is said to be persistence if there exist numbers $m$ and $M$ with $0<m$ $\leq M<\infty$ such that for any initial $x_{-k}, x_{-k+1}, \cdots, x_{0} \in(0, \infty)$ there exists a positive integer $N$ which depends on the initial conditions such that $m \leq x_{n} \leq M$ for all $n \geq N$.

Definition (1.3) (Stability)

Let $I$ be some interval of real numbers.

1) The equilibrium point $\bar{x}$ of Equation (2) is locally stable if for every $\varepsilon>0$, there exists $\delta>0$ such that for $x_{-k}, x_{-k+1}, \cdots, x_{0} \in I$ with

$$
\left|x_{-k}-\bar{x}\right|+\left|x_{-k+1}-\bar{x}\right|+\cdots+\left|x_{0}-\bar{x}\right|<\delta,
$$

we have $\left|x_{0}-\bar{x}\right|<\varepsilon$ for all $n \geq-k$.

2) The equilibrium point $\bar{x}$ of Equation (2) is locally asymptotically stable if $\bar{x}$ is locally stable solution of Equation (2) and there exists $\gamma>0$, such that for all $x_{-k}, x_{-k+1}, \cdots, x_{0} \in I$ with

$$
\left|x_{-k}-\bar{x}\right|+\left|x_{-k+1}-\bar{x}\right|+\cdots+\left|x_{0}-\bar{x}\right|<\delta,
$$

we have $\lim _{n \rightarrow \infty} x_{n}=\bar{x}$.

3) The equilibrium point $\bar{x}$ of Equation (2) is global attractor if for all $x_{-k}, x_{-k+1}, \cdots, x_{0} \in I$, we have $\lim _{n \rightarrow \infty} x_{n}=\bar{x}$.

4) The equilibrium point $\bar{x}$ of Equation (2) is globally asymptotically stable if $\bar{x}$ is locally stable, and $\bar{x}$ is also a global attractor of Equation (2).

5) The equilibrium point $\bar{x}$ of Equation (2) is unstable if $\bar{x}$ not locally stable.

The linearized equation of Equation (2) about the equi- librium $\bar{x}$ is the linear difference equation

$$
y_{n+1}=\sum_{i=0}^{k}\left(\partial F(\bar{x}, \bar{x}, \cdots, \bar{x}) /\left(\partial x_{n-i}\right)\right) y_{n-i}
$$

Theorem (1.4) [10] Assume that $p, q \in \mathfrak{R}$ (real numbers) and $k \in\{0,1,2, \cdots\}$ Then

$$
|p|+|q|<1
$$

is a sufficient condition for the asymptotic stability of the difference equation

$$
x_{n+1}+p x_{n}+q x_{n-k}=0, n=0,1, \cdots .
$$

Remark (1.5) Theorem (1.4) can be easily extended to a general linear equations of the form

$$
x_{n+k}+p_{1} x_{n+k-1}+\cdots+p_{k} x_{n}=0, n=0,1, \cdots
$$

where $p_{1}, p_{2}, \cdots, p_{k} \in \mathfrak{R}$ (real numbers) and $k \in\{1,2 \cdots\}$. Then Equation (4) is asymptotically stable provided that $\sum_{i=1}^{k}\left|p_{i}\right|<1$.

\section{Definition (1.6) (Periodicity)}

A sequence $\left\{x_{n}\right\}_{n=-k}^{\infty}$ is said to be periodic with pe$\operatorname{riod} p$ if $x_{n+p}=x_{n}$ for all $n \geq-k$.

\section{Solution and Periodicity}

In this section we give a specific form of the solutions of the difference Equation (1).

\section{Theorem (2.1)}

Let $\left\{x_{n}\right\}_{n=-k}^{\infty}$ be a solution of Equation (1). Then Equation (1) have all solutions and the solutions are

$$
\begin{aligned}
& x_{14 n-5}=k, x_{14 n-4}=h, x_{14 n-3}=L, x_{14 n-2}=\alpha, \\
& x_{14 n-1}=\beta, x_{14 n}=\gamma, x_{14 n+1}=(\gamma \alpha h) /(\beta L k), \\
& x_{14 n+2}=1 / k, x_{14 n+3}=1 / h, x_{14 n+4}=1 / L, x_{14 n+5}=1 / \alpha, \\
& x_{14 n+6}=1 / \beta, x_{14 n+7}=1 / \gamma, x_{14 n+8}=\beta L k / \gamma \alpha h
\end{aligned}
$$

where $x_{-5}=k, x_{-4}=h, x_{-3}=L, x_{-2}=\alpha, x_{-1}=\beta, x_{0}=\gamma$.

Proof:

For $n=0$ the result holds. Now suppose that $n>0$ and that our assumption holds for $n-1$. We shall show that the result holds for $n$. By using our assumption for $n-1$, we have the following:

$$
\begin{aligned}
& x_{14 n-19}=k, x_{14 n-18}=h, x_{14 n-17}=L, x_{14 n-16}=\alpha, \\
& x_{14 n-15}=\beta, x_{14 n-14}=\gamma, x_{14 n-13}=(\gamma \alpha h) /(\beta L k), \\
& x_{14 n-12}=1 / k, x_{14 n-11}=1 / h, x_{14 n-10}=1 / L, x_{14 n-9}=1 / \alpha \\
& x_{14 n-8}=1 / \beta, x_{14 n-7}=1 / \gamma, x_{14 n-6}=\beta L k / \gamma \alpha h
\end{aligned}
$$

Now, it follows from Equation (1) that

$$
\begin{aligned}
x_{14 n-5} & =x_{14 n-6} x_{14 n-8} x_{14 n-10} /\left(x_{14 n-7} x_{14 n-9} x_{14 n-11}\right) \\
& =(\beta L k / \gamma \alpha h)(1 / \beta)(1 / L) /(1 l \gamma)(1 / \alpha)(1 / h)=k
\end{aligned}
$$




$$
\begin{aligned}
& x_{14 n-4}=x_{14 n-5} x_{14 n-7} x_{14 n-9} /\left(x_{14 n-6} x_{14 n-8} x_{14 n-10}\right)=h \\
& x_{14 n-3}=x_{14 n-4} x_{14 n-6} x_{14 n-8} /\left(x_{14 n-5} x_{14 n-7} x_{14 n-9}\right)=L \\
& x_{14 n-2}=x_{14 n-3} x_{14 n-5} x_{14 n-7} /\left(x_{14 n-4} x_{14 n-6} x_{14 n-8}\right)=\alpha
\end{aligned}
$$

similarly we can derive,

$$
\begin{aligned}
& x_{14 n-2}=\alpha, x_{14 n}=\gamma, \\
& x_{14 n+1}=(\gamma \alpha h) /(\beta L k), x_{14 n+2}=1 / k, \\
& x_{14 n+3}=1 / h, x_{14 n+4}=1 / L, x_{14 n+5}=1 / \alpha, \\
& x_{14 n+6}=1 / \beta, x_{14 n+7}=1 / \gamma, x_{14 n+8}=\beta L k / \gamma \alpha h .
\end{aligned}
$$

Thus, the proof is completed.

Theorem (2.2)

Suppose that $\left\{x_{n}\right\}_{n=-k}^{\infty}$ be a solution of Equation (1). Then all solutions of Equation (1) are periodic with period fourteen.

\section{Proof:}

From Equation (1), we see that

$$
\begin{aligned}
& x_{n+1}=\left(x_{n} x_{n-2} x_{n-4}\right) /\left(x_{n-1} x_{n-3} x_{n-5}\right) \\
& x_{n+2}=\left(x_{n+1} x_{n-1} x_{n-3}\right) /\left(x_{n} x_{n-2} x_{n-4}\right)=1 / x_{n-5} \\
& x_{n+3}=\left(x_{n+2} x_{n} x_{n-2}\right) /\left(x_{n+1} x_{n-1} x_{n-3}\right)=1 / x_{n-4} \\
& x_{n+4}=\left(x_{n+3} x_{n+1} x_{n-1}\right) /\left(x_{n+2} x_{n} x_{n-2}\right)=1 / x_{n-3} \\
& x_{n+5}=1 / x_{n-2} \& x_{n+6}=1 / x_{n-1} \& x_{n+7}=1 / x_{n} \\
& x_{n+8}=1 / x_{n+1} \& x_{n+9}=1 / x_{n+2}=x_{n-5} \& x_{n+10}=x_{n-4} \\
& x_{n+11}=x_{n-3} \& x_{n+12}=x_{n-2} \& x_{n+13}=x_{n-1} \& x_{n+14}=x_{n}
\end{aligned}
$$

which completes the proof.

\section{Stability of Solutions}

In this section we study the local stability of the solutions of Equation (1).

\section{Lemma (3.1)}

Equation (1) have two equilibrium points which are 0 and 1 .

\section{Proof:}

For the equilibrium points of Equation (1), we can write

$$
\bar{x}=\bar{x} \cdot \bar{x} \cdot \bar{x} /(\bar{x} \cdot \bar{x} \cdot \bar{x})
$$

Then $\bar{x}^{4}=\bar{x}^{3}$, i.e. $\bar{x}^{4}-\bar{x}^{3}=0$

Thus the equilibrium points of Equation (1) is are 0 and 1 .

\section{Theorem (3.2)}

The equilibrium points $\bar{x}=0$ and $\bar{x}=1$ are unstable.

\section{Proof:}

We will prove the theorem at the equilibrium point $\bar{x}=1$ and the proof at the equilibrium point $\bar{x}=0$ by the same way.

Let $f:(0, \infty)^{6} \rightarrow(0, \infty)$ be a continuous function defined by

$$
f\left(u_{1}, u_{2}, u_{3}, u_{4}, u_{5}, u_{6}\right)=\left(u_{1} u_{3} u_{5}\right) /\left(u_{2} u_{4} u_{6}\right)
$$

Therefore it follows that

$$
\begin{gathered}
\partial f / \partial u_{1}=\left(u_{3} u_{5}\right) /\left(u_{2} u_{4} u_{6}\right) \\
\partial f / \partial u_{2}=0-\left(u_{1} u_{3} u_{5}\right)\left(u_{4} u_{6}\right) /\left(u_{2} u_{4} u_{6}\right)^{2} \\
=-\left(u_{1} u_{3} u_{5}\right) /\left(u_{2}^{2} u_{4} u_{6}\right) . \\
\partial f / \partial u_{3}=\left(u_{1} u_{5}\right) /\left(u_{2} u_{4} u_{6}\right) \\
\partial f / \partial u_{4}=-\left(u_{1} u_{3} u_{5}\right) /\left(u_{2} u_{4}^{2} u_{6}\right) \\
\partial f / \partial u_{5}=\left(u_{1} u_{3}\right) /\left(u_{2} u_{4} u_{6}\right) \\
\partial f / \partial u_{6}=-\left(u_{1} u_{3} u_{5}\right) /\left(u_{2} u_{4} u_{6}^{2}\right)
\end{gathered}
$$

At the equilibrium point $\bar{x}=1$ we have

$$
\begin{aligned}
& \partial f / \partial u_{1}=1=p_{1}, \quad \partial f / \partial u_{2}=-1=p_{2}, \\
& \partial f / \partial u_{3}=1=p_{3}, \quad \partial f / \partial u_{4}=-1=p_{4}, \\
& \partial f / \partial u_{5}=1=p_{5}, \quad \partial f / \partial u_{6}=-1=p_{6}
\end{aligned}
$$

Then the linearized equation of Equation (1) about $\bar{x}=1$ is

i.e.

$$
\begin{aligned}
y_{n+1} & -p_{1} y_{n}-p_{2} y_{n-1}-p_{3} y_{n-2}-p_{4} y_{n-3} \\
& -p_{5} y_{n-4}-p_{6} y_{n-5}=0
\end{aligned}
$$

$$
y_{n+1}-y_{n}+y_{n-1}-y_{n-2}+y_{n-3}-y_{n-4}+y_{n-5}=0
$$

Whose characteristic equation is

$$
\lambda^{6}-\lambda^{5}+\lambda^{4}-\lambda^{3}+\lambda^{2}-\lambda+1=0
$$

By the generalization of theorem (1.4) we have

$$
|1|+|-1|+|1|+|1|+|-1|+|1|<1
$$

which is impossible. This means that the equilibrium point $\bar{x}=1$ is unstable. Similarly, we can see that the equilibrium point $\bar{x}=0$ is unstable.

\section{Numerical Examples}

For confirming the results of this section, we consider numerical examples which represent different types of solutions to Equation (1).

\section{Example 4.1}

Consider $x_{-5}=1, x_{-4}=2, x_{-3}=4, x_{-2}=-1, x_{-1}=-2$, and $x_{0}=7$. See Figure 1 .

\section{Example 4.2}

Consider $x_{-5}=1, x_{-4}=-1, x_{-3}=2, x_{-2}=-6, x_{-1}=5$, and $x_{0}=-4$. See Figure 2 .

\section{Example 4.3}

Consider $x_{-5}=3, x_{-4}=5, x_{-3}=-7, x_{-2}=-3, x_{-1}=2$, and $x_{0}=-1$. See Figure 3 .

\section{Example 4.4}

Consider $x_{-5}=-4, x_{-4}=3, x_{-3}=-2, x_{-2}=9, x_{-1}=17$, 


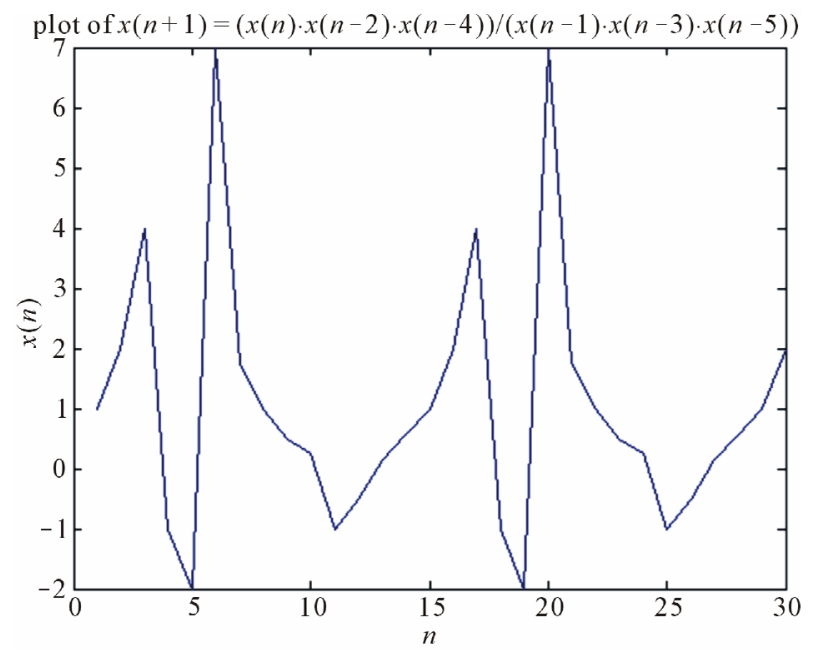

Figure 1. The periodicity of solutions with period 14 with unstable equilibrium points $\bar{x}=1$ and $\bar{x}=0$.

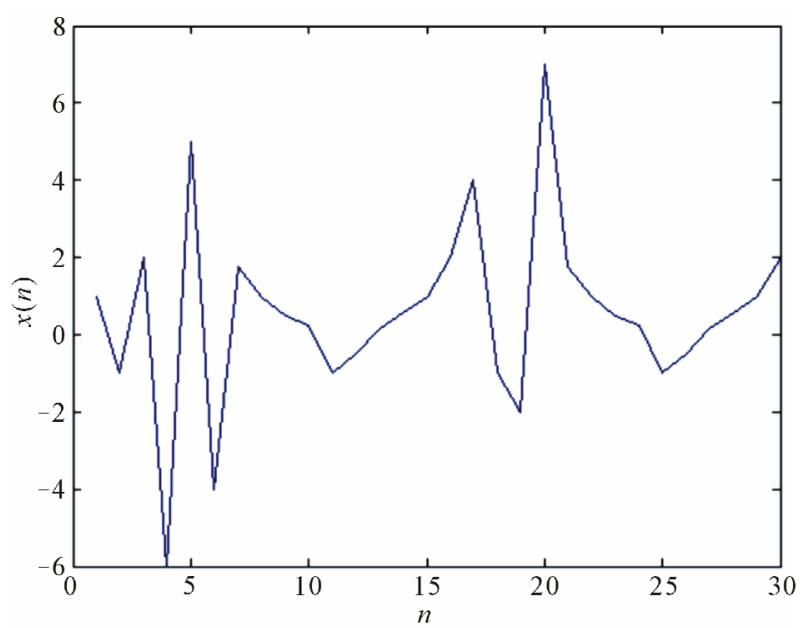

Figure 2. Periodicity of solutions with period 14 with unstable equilibrium points $\bar{x}=1, \bar{x}=0$.

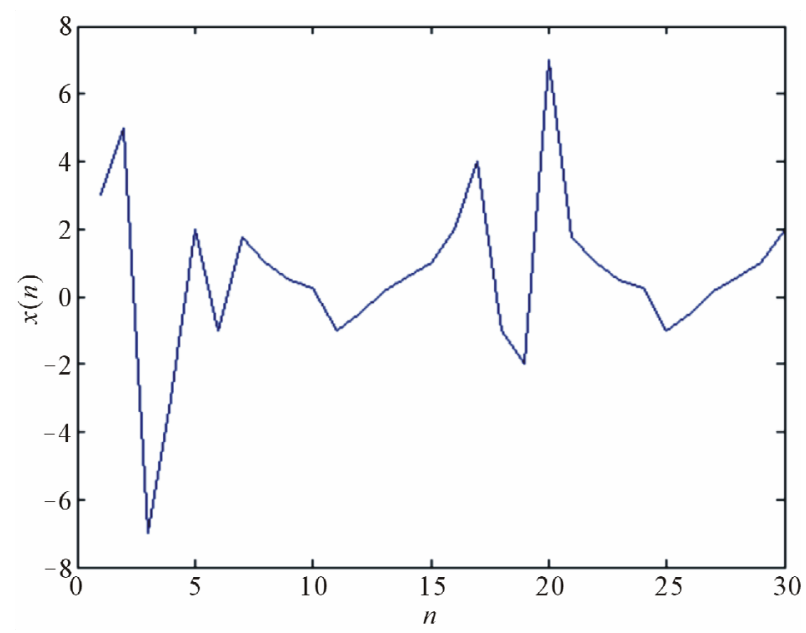

Figure 3. Periodicity of solutions with period 14 with unstable equilibrium points $\bar{x}=1$ and $\bar{x}=0$.

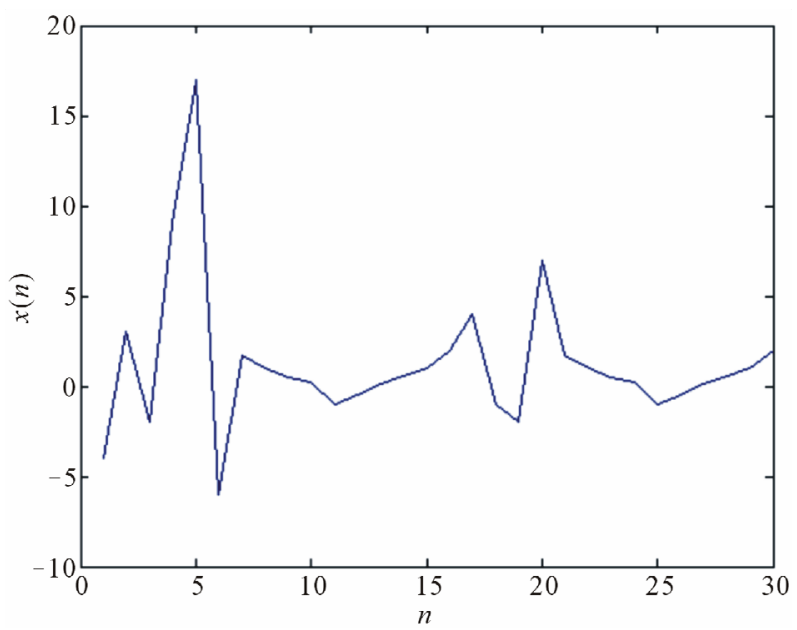

Figure 4. The periodicity of solutions with period 14 with unstable equilibrium points $\bar{x}=1, \bar{x}=0$.

and $x_{0}=-6$. See Figure 4 .

\section{Acknowledgements}

We want to thank the referee for his useful suggestions.

\section{REFERENCES}

[1] C. Cinar, "On the Positive Solutions of the Difference Equation $x_{n+1}=x_{n-1} /\left(-1+a x_{n} x_{n-1}\right)$," Applied Mathematics and Computation, Vol. 158, No. 3, 2004, pp. 793-797. doi:10.1016/j.amc.2003.08.139

[2] C. Cinar, "On the Positive Solutions of the Difference Equation $x_{n+1}=a x_{n-1} /\left(1+b x_{n} x_{n-1}\right)$," Applied Mathematics and Computation, Vol. 156, No. 2, 2004, pp. 587-590. doi:10.1016/j.amc.2003.08.010

[3] S. N. Elaydi, "An Introduction to Difference Equations," Springer-Verlag Inc., New York, 1996.

[4] R. Karatas, C. Cinar and D. Simsek, "On Positive Solutions of the Difference Equation $x_{n+1}=x_{n-5} /\left(1+x_{n-2} x_{n-5}\right)$," International Journal of Contemporary Mathematical Sciences, Vol. 1, No. 10, 2006, pp. 495-500.

[5] T. F. Ibrahim, "On the Third Order Rational Difference Equation $x_{n+1}=\left(x_{n} x_{n-2}\right) /\left(x_{n-1}\left(\alpha+\beta x_{n} x_{n-2}\right)\right)$," International Journal of Contemporary Mathematical Sciences, Vol. 4, No. 27, 2009, pp. 1321-1334.

[6] T. F. Ibrahim, "Global Asymptotic Stability of a Nonlinear Difference Equation with Constant Coefficients," Mathematical Modelling and Applied Computing, Vol. 1, No. 1, 2009.

[7] T. F. Ibrahim, "Dynamics of a Rational Recursive Sequence of Order Two," International Journal of Mathematics and Computation, Vol. 5, No. D09, 2009, pp. 98105.

[8] T. F. Ibrahim, "Solvability and Attractivity of the Solu- 
tions of a Rational Difference Equation," Journal of Pure and Applied Mathematics: Advances and Applications, Vol. 2, No. 2, 2009, pp. 227-237.

[9] T. F. Ibrahim, "Periodicity and Analytic Solution of a Recursive Sequence with Numerical Examples," Journal of Interdisciplinary Mathematics, Vol. 12, No. 5, 2009, pp. 701-708.

[10] V. L. Kocic and G. Ladas, "Global Behavior of Nonlinear Difference Equations of Higher Order with Applications," Kluwer Academic Publishers, Dordrecht, 1993.

[11] M. R. S. Kulenovic and G. Ladas, "Dynamics of Second Order Rational Difference Equations with Open Problems and Conjectures," Chapman \& Hall/CRC Press, Boca
Raton, 2001. doi:10.1201/9781420035384

[12] G. Ladas and M. Kulenovic, "On Period Two Solutions of $x_{n+1}=\left(\alpha+\beta x_{n}+\gamma x_{n-1}\right) /\left(A+B x_{n}+C x_{n-1}\right)$," Journal of Difference Equations and Applications, Vol. 6, 2000, pp. 641-646.

[13] D. Simsek, C. Cinar and I. Yalcinkaya, "On the Recursive Sequence $x_{n+1}=x_{n-3} /\left(1+x_{n-1}\right)$," International Journal of Contemporary Mathematical Sciences, Vol. 1, No. 10, 2006, pp. 475-480. 